\title{
Subclinical Hypothyroidism and features of metabolic syndrome in Saudi elderly women
}

\author{
Eman M Alissa(1), Gordon A Ferns(2)
}

(1) Faculty of Medicine, King AbdulAziz University, KSA

(2) Medical Education and Metabolic Medicine, Brighton and Sussex Medical School, University of Brighton, UK

Topic: Obesity and cardiovascular endocrinology, e-Poster number is EP-542

\section{OBJECTIVES}

Metabolic syndrome (MetS) is

characterized by a clustering of metabolic features that include central obesity, impaired glucose tolerance, dyslipidemia and hypertension. Individuals with MetS are at increased risk for diabetes mellitus (DM) and cardiovascular disease (CVD). The impact of thyroid hormone deficiency on glucose and insulin metabolism is not well understood. However, thyroid hormones play a role in lipid synthesis, metabolism and mobilization. Sub-clinical hypothyroidism $(\mathrm{SCH})$ has been proposed to be a risk factor for low-grade inflammation, CVD, hypertension, and hyperlipidemia. Metabolic syndrome is a condition in which there are features that overlap those of hypothyroidism

Aim: To investigate the frequency of $\mathrm{SCH}$ in adults with MetS. To assess whether MetS features were associated with $\mathrm{SCH}$.

\section{METHODS}

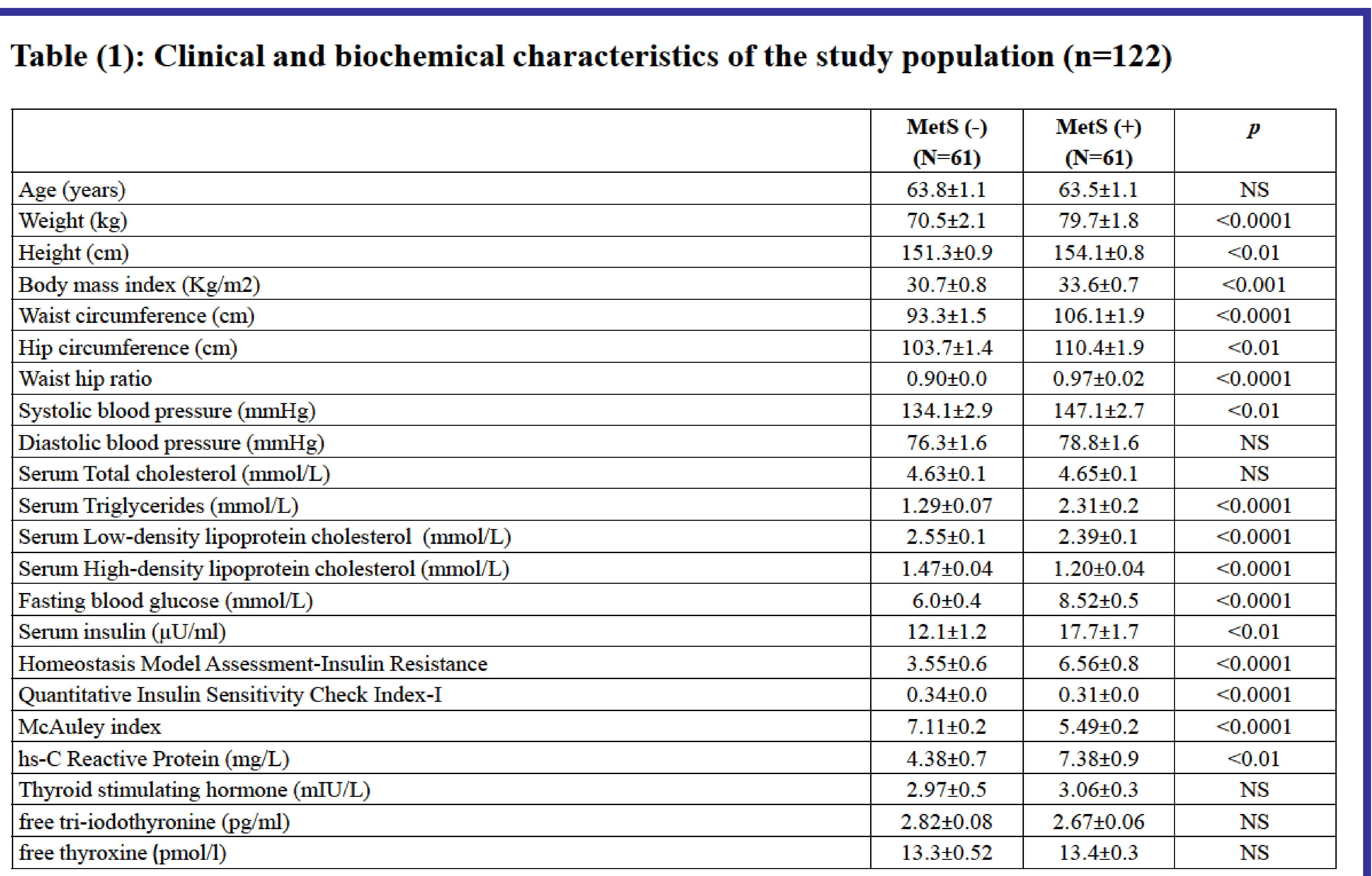

Data are expressed as means $₫$ standard errors. Variables are compared by Mann-Whitney test
Table (2): Association between thyroid stimulating hormone

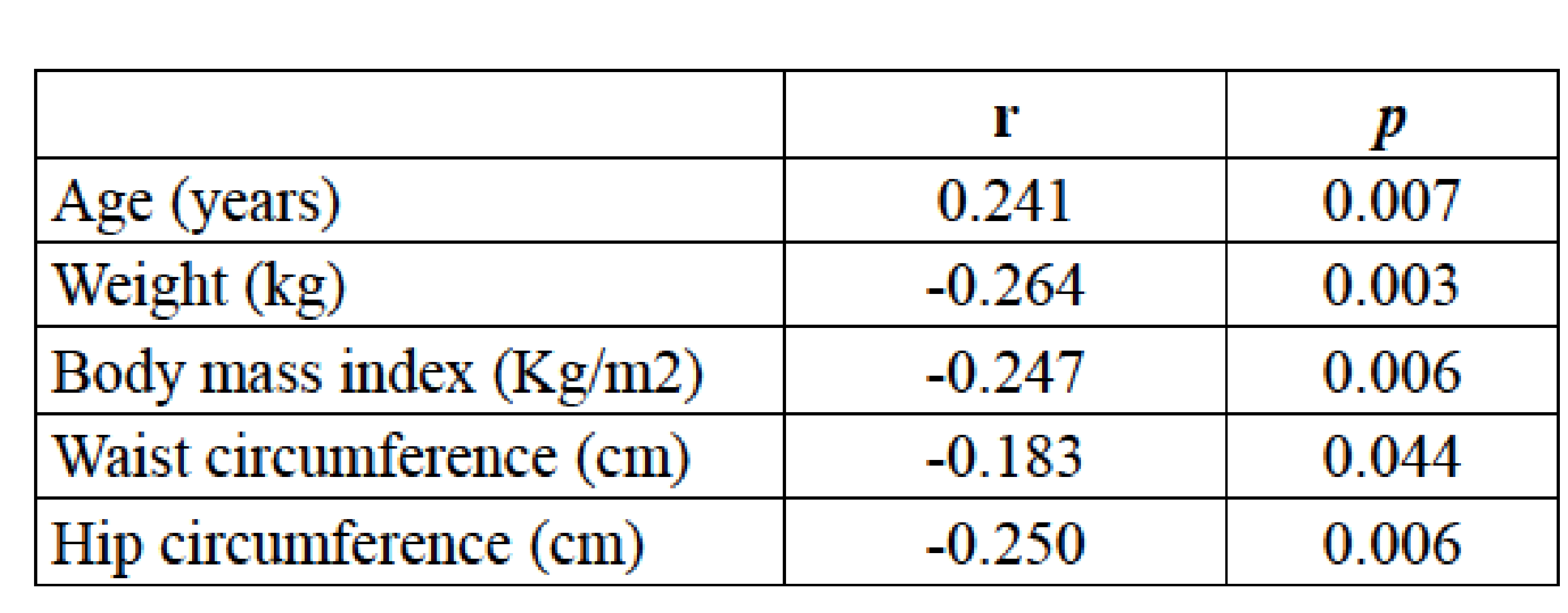
and metabolic risk factors in the study population $(n=122)$

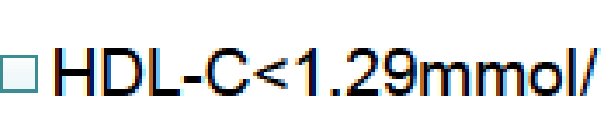

$\square T G \geq 1.7 \mathrm{mmol} / \mathrm{L}$

$\square \mathrm{FBG} \geq 5.6 \mathrm{mmol} / \mathrm{L}$

$\square \mathrm{BP} \geq 130 / 85 \mathrm{mmHg}$ $\square W C>88 \mathrm{~cm}$

\section{RESULTS}

Clinical and biochemical characteristics of the study population are shown in Table (1). The prevalence of SCH was $26 \%$ in the MetS group and $15 \%$ in the control group ( $p>0.05)$. Although the two disease entities had some common biochemical markers, there were no significant relationships. The proportions of central obesity, impaired fasting glucose, hypertension,

hypertriglyceridemia, and low HDL-C were $80 \%, 48 \%, 52 \%, 36 \%$, and $36 \%$ in subjects with SCH (Figure 1). Table (2) shows the correlation of TSH levels with the variables related to CVD and MetS. The prevalence of SCH increased with age $(r=0.241$, $\mathrm{p}<0.01)$. All obesity measures were consistently inversely associated with serum TSH level.

Subjects with MetS had significantly higher levels of blood pressure, fasting blood glucose, fasting blood profile (except for HDL-C which was significantly lower), and serum insulin than their control counterparts $(p<0.01)$. All anthropometric measurements and insulin resistance measures were lower in the control group than in MetS patients $(p<0.01)$. CRP was higher in the MetS patients than their matching controls $(p<0.01)$.

\section{CONCLUSIONS}

$\mathrm{SCH}$ is increased in patients with MetS, therefore hypothyroidism should be considered in newly diagnosed MetS patients. Of all MetS components, waist circumference was the only component negatively associated with serum TSH levels. Low-grade inflammation was more prevalent among the National Cholesterol Education Program-defined MetS patients than their age-matched controls.

\section{References}

Grundy SM, Cleeman JI, Daniels SR, Donato KA, Eckel RH, Franklin BA, et al. Diagnosis and management of the metabolic syndrome: an American Heart Association/National Heart, Lung, and Blood Institute scientific statement: Executive Summary. Crit Pathw Cardiol. 2005;4(4):198-203

Jones DD, May KE, Geraci SA. Subclinical thyroid diseases. Am J Med 2010;123:502-504. 